Claremont Colleges

Scholarship@ Claremont

All HMC Faculty Publications and Research

HMC Faculty Scholarship

11-1-1999

\title{
Recounting Fibonacci and Lucas Identities
}

Arthur T. Benjamin

Harvey Mudd College

Jennifer J. Quinn

Occidental College

\section{Recommended Citation}

Benjamin, Arthur T. and Jennifer J. Quinn. "Recounting Fibonacci and Lucas Identities." The College Mathematics Journal, Vol 30, No. 5, pp. 359-366, November, 1999.

This Article is brought to you for free and open access by the HMC Faculty Scholarship at Scholarship @ Claremont. It has been accepted for inclusion in All HMC Faculty Publications and Research by an authorized administrator of Scholarship @ Claremont. For more information, please contact scholarship@cuc.claremont.edu. 
Recounting Fibonacci and Lucas Identities

Author(s): Arthur T. Benjamin and Jennifer J. Quinn

Source: The College Mathematics Journal, Vol. 30, No. 5 (Nov., 1999), pp. 359-366

Published by: Mathematical Association of America

Stable URL: http://www.jstor.org/stable/2687539

Accessed: 10/06/2013 17:31

Your use of the JSTOR archive indicates your acceptance of the Terms \& Conditions of Use, available at http://www.jstor.org/page/info/about/policies/terms.jsp

JSTOR is a not-for-profit service that helps scholars, researchers, and students discover, use, and build upon a wide range of content in a trusted digital archive. We use information technology and tools to increase productivity and facilitate new forms of scholarship. For more information about JSTOR, please contact support@jstor.org. 


\title{
Recounting Fibonacci and Lucas Identities
}

\author{
Arthur T. Benjamin and Jennifer J. Quinn
}

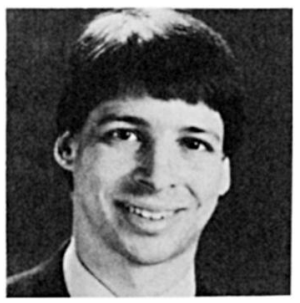

Arthur Benjamin (benjamin@math.hmc.edu) earned his B.S. in Applied Mathematics from Carnegie Mellon and his Ph.D. in Mathematical Sciences from Johns Hopkins where he studied discrete optimization under Alan J. Goldman. Since 1989, he has taught at Harvey Mudd College, where he is currently an associate professor. $\mathrm{He}$ is editor of the Spectrum book series for MAA, and an Associate Editor of Mathematics Magazine. He was recently awarded the MAA's Haimo Award for distinguished college teaching.

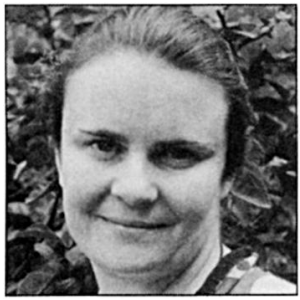

Jennifer Quinn (jquinn@oxy.edu) is an Associate Professor of Mathematics at Occidental College in Los Angeles. She earned her degrees from Williams College, University of Illinois, Chicago, and the University of Wisconsin, Madison continually moving the "Quinn family center of gravity" west. Her primary research interests are combinatorics and graph theory. Lately she has been having great fun hosting The Number Years, a mathematical game show co-created with Occidental graduate Eric Libicki and Art Benjamin.

Behold, the Fibonacci numbers $0,1,1,2,3,5,8,13,21,34,55, \ldots$ defined recursively as $F_{0}=0, F_{1}=1$, and for $n$ greater than or equal to $2, F_{n}=F_{n-1}+F_{n-2}$. This sequence of numbers has intrigued and inspired mathematicians for centuries $[10,11]$. Less well-known but close companions to the Fibonacci numbers are the Lucas numbers $2,1,3,4,7,11,18,29,47,76,123, \ldots$ which satisfy the same recurrence relation starting with different initial conditions. Here $L_{0}=2, L_{1}=1$, and for $n$ greater than or equal to $2, L_{n}=L_{n-1}+L_{n-2}$.

Pattern seekers are drawn to the connections within and between these two number sequences. In this paper, we will explore some of our favorites:

$$
\begin{aligned}
F_{m+n} & =F_{m+1} F_{n}+F_{m} F_{n-1} \\
L_{m+n} & =F_{m+1} L_{n}+F_{m} L_{n-1} \\
F_{n-1}+F_{n+1} & =L_{n} \\
L_{n-1}+L_{n+1} & =5 F_{n} \\
F_{n} L_{n} & =F_{2 n} \\
F_{n}^{2} & =F_{n-1} F_{n+1}+(-1)^{n-1}
\end{aligned}
$$

All of these identities can be proved using algebraic means (induction, generating functions, determinants, hyperbolic functions, and so on) [10]. While these techniques are valid, they can be less than illuminating. In contrast, combinatorial arguments view an identity as a story which can be told from two different points of view. Each side recounts the story in a different but accurate way. 
We present several combinatorial interpretations of Fibonacci and Lucas numbers and their connections. Using one of these interpretations (tiling), many Fibonacci and Lucas identities practically reduce to "proofs without words" [7]. For your enjoyment, we present our favorites in a concrete combinatorial fashion.

\section{Combinatorial Interpretations}

We begin with different interpretations of Fibonacci numbers.

Let $a_{n}$ denote the number of length $n$ binary sequences with no consecutive 0 's. Then $a_{n}$ satisfies the recurrence $a_{n}=a_{n-1}+a_{n-2}$ since any such sequence beginning with 1 can be extended in $a_{n-1}$ ways and a sequence beginning with 0 must be followed by 1 and can be extended in $a_{n-2}$ ways. Thus $a_{n}$ grows like the Fibonacci numbers. Since $a_{0}=1=F_{2}$ and $a_{1}=2=F_{3}$, we have $a_{n}=F_{n+2}$ for all $n$ greater than zero.

For a graph theoretic interpretation of Fibonacci numbers, let $b_{n}$ denote the number of ways to select a subset of nonadjacent vertices from a path on $n$ vertices (Figure 1). Such a subset of vertices is called an independent set. Notice $b_{n-2}$ and $b_{n-1}$ count the number of independent subsets that do and do not contain the first point on the path, respectively. Thus $b_{n}$ also satisfies the Fibonacci recurrence. Again $b_{0}=1$ and $b_{1}=2$, so $b_{n}=F_{n+2}=a_{n}$ for $n$ greater than or equal to zero. A natural correspondence exists between these two representations. Independent sets of vertices correspond to the 0's in the binary sequences. The selected subset from Figure 1 will correspond to the sequence 0110101101.

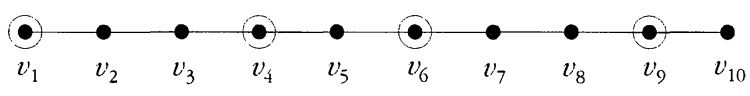

Figure 1. A path on 10 vertices with independent vertex set $v_{1}, v_{4}, v_{6}$, and $v_{9}$.

If we let $c_{n}$ denote the number of series of 1's and 2's that add to $n$, then $c_{1}=1$ and $c_{2}=2$. By conditioning on the first number in our sum, we see that $c_{n}=c_{n-1}$ $+c_{n-2}$. Therefore, $c_{n}=F_{n+1}$. There is a natural correspondence between $c_{n+1}$ and $b_{n}$. For a given series of 1's and 2's that add to $n+1$, associate the subset of vertices whose indices are not partial sums of the series. For example, the series $2+1+2$ $+2+1+2+1=11$ has partial sums $2,3,5,7,8,10$, and 11, yielding the independent set $v_{1}, v_{4}, v_{6}, v_{9}$ in Figure 1 .

Our primary focus will be on the number of ways to tile an $n$-board, a $1 \times n$ checkerboard with cells labelled $1,2, \ldots, n$. Let $f_{n}$ denote the number of ways to tile an $n$-board with $1 \times 1$ squares and $1 \times 2$ dominoes. Associating each square with a 1 , and each domino with a 2, we see $f_{n}=c_{n}$. See Figure 2. Hence,

$$
f_{n}=F_{n+1}
$$

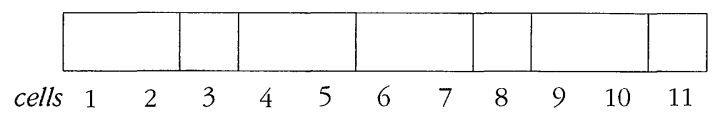

Figure 2. The tiling associated with the series $2+1+2+2+1+2+1=11$. 
This representation leads to elegant combinatorial interpretations of Fibonacci number identities. For example, identity (1) (after replacing $n$ with $n+1$ ) becomes

$$
f_{m+n}=f_{m} f_{n}+f_{m-1} f_{n-1} .
$$

The left side of this identity counts the number of ways to tile a length $m+n$ board. To interpret the right side, we notice that tilings come in two varieties: either they can be separated into a length $m$ tiling followed by a length $n$ tiling, or they cannot. There are $f_{m} f_{n}$ tilings of the first type. Tilings of the second type must contain a domino covering cells $m$ and $m+1$. The remaining board can be covered $f_{m-1} f_{n-1}$ ways.

Combinatorial identities can be generated by conditioning on different events. The previous proof conditions on whether or not a tiling can be separated immediately following the $m$ th cell. The identity

$$
f_{n}=\sum_{k=0}^{\lfloor n / 2\rfloor}\left(\begin{array}{c}
n-k \\
k
\end{array}\right)
$$

simply reflects the fact that the number of tilings of an $n$-board using exactly $k$ dominoes (and hence $n-2 k$ squares) is $\left(\begin{array}{c}(n-2 k)+k \\ k\end{array}\right)$. For other tiling proofs of Fibonacci identities, see $[\mathbf{2}, \mathbf{3}, \mathbf{5}, \mathbf{6}]$.

Now we turn our attention to Lucas numbers. As was shown in [8] and [9], Lucas numbers act just like Fibonacci numbers running in circles.

Let $A_{n}$ denote the number of length $n$ circular binary sequences with no consecutive 0's (see Figure 3). The length 2 and 3 circular sequences are 01, 10, 11, 011, 101, 110, and 111. So $A_{2}=3=L_{2}$ and $A_{3}=4=L_{3}$. To prove that $A_{n}=L_{n}$, we show that $A_{n}$ satisfies the same recurrence relation. We condition on the first digit. A circular sequence beginning with 1 can be completed in $a_{n-1}=F_{n+1}$ ways. A 0 must be surrounded by 1's, so that a circular sequence beginning with 0 can be completed in $a_{n-3}=F_{n-1}$ ways. Thus $A_{n}=F_{n+1}+F_{n-1}$, which obviously satisfies the desired recurrence. Hence for $n \geq 2, A_{n}=L_{n}$ and identity (3) is proved along the way.

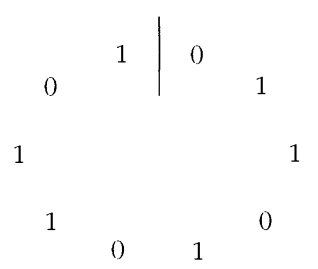

Figure 3. The circular binary sequence 0110101101 with no consecutive 0's.

Analogously, let $B_{n}$ denote the number of independent sets in a cycle graph with $n$ vertices. Just as before, $B_{n}=A_{n}=L_{n}$, since independent sets of vertices correspond to the 0's in the binary sequences. This is illustrated in Figure 4.

Let $C_{n}$ denote the number of series of 1's and 2's that sum to $n$ with the end point restriction that it may not begin and end with a 2 . By conditioning on the first term, we obtain $C_{n}=c_{n-1}+c_{n-3}=F_{n}+F_{n-2}=L_{n-1}$.

As you might expect, we let $l_{n}$ denote the number of ways to tile a circular $1 \times n$ board with squares and dominoes (objects of length 1 and 2 respectively). Cells are 


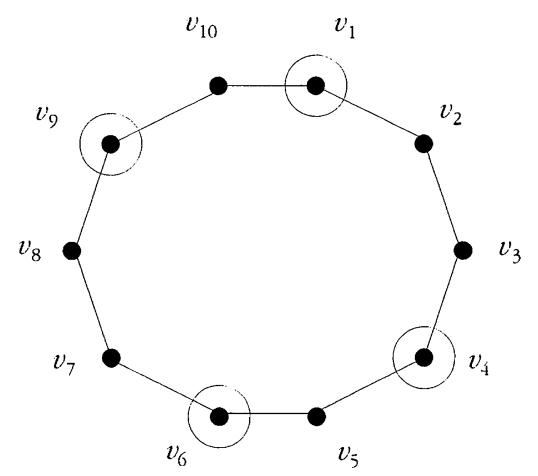

Figure 4. Cycle on 10 vertices with independent set $v_{1}, v_{4}, v_{6}$, and $v_{9}$ corresponding to the circular sequence given in Figure 3.

labelled 1 through $n$ and a tiling is called an $n$-bracelet. See Figure 5 . An $n$-bracelet is out of phase if the same domino covers cells $n$ and 1 . Otherwise the $n$-bracelet is in phase. The number of in phase $n$-bracelets is $f_{n}=F_{n+1}$ and the number of out of phase $n$-bracelets is $f_{n-2}=F_{n-1}$. Hence $l_{n}=F_{n+1}+F_{n-1}=L_{n}$.

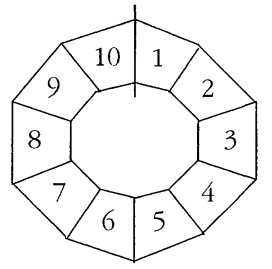

Circular 10-board

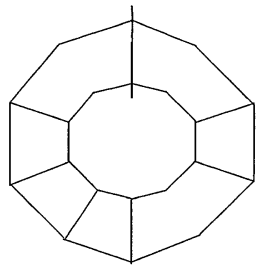

In phase

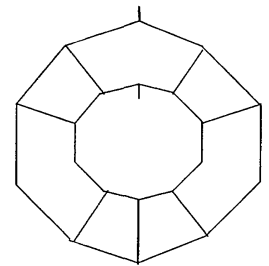

Out of phase

Figure 5. Illustrations of a circular 10-board, an in phase 10-bracelet and an out of phase 10-bracelet.

Using this interpretation, we provide a combinatorial proof for identity (2), equivalent to

$$
l_{m+n}=f_{m} l_{n}+f_{m-1} l_{n-1} .
$$

For a given bracelet, we say the break point occurs along the right edge of the tile covering cell 1 . To prove the above identity, we partition the $l_{m+n}(m+n)$-bracelets into two cases: those that have a length $m$ string of tiles immediately following the break point, and those that do not. The number of tilings in the first case is $l_{n} f_{m}$ (start with an $n$-bracelet and insert a length $m$ string after its break point). The remaining $(m+n)$-bracelets are obtained by taking an $(n-1)$-bracelet and inserting a length $m-1$ string plus a domino after its break point. There are $l_{n-1} f_{m-1}$ bracelets of this type.

Here's a quick proof of identity (5), equivalent to

$$
f_{n-1} l_{n}=f_{2 n-1} \text {. }
$$

For every tiling of a $(2 n-1)$-board we generate a unique pair $(T, B)$ where $T$ is an $(n-1)$-board tiling and $B$ is an $n$-bracelet. If cells $n$ and $n+1$ are covered by a domino $D$, let $T$ be created by the tiles covering cells 1 through $n-1$ and let $B$ 

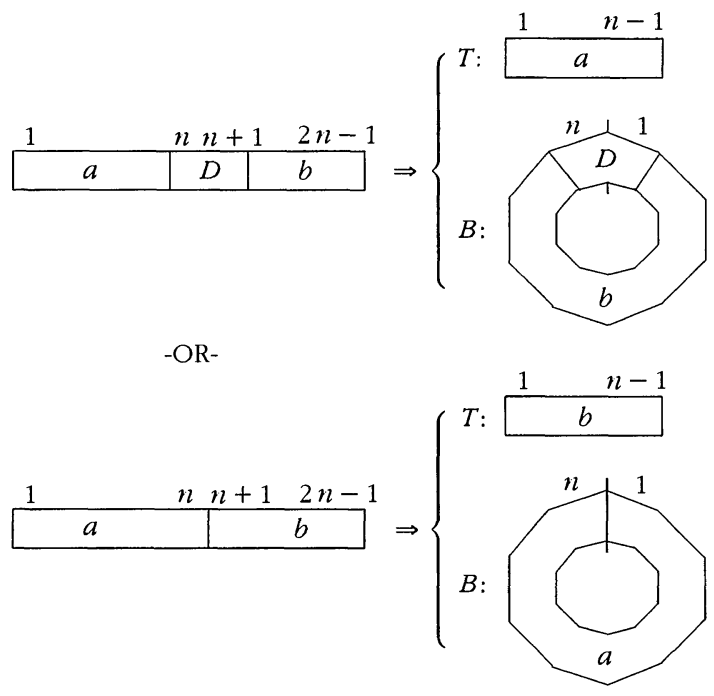

Figure 6. Combinatorial proof that $f_{2 n-1}=f_{n-1} l_{n}$.

be the out of phase bracelet created from the remaining tiles, placing $D$ over cells $n$ and 1 . See Figure 6 . Otherwise let $T$ be created by the tiles covering cells $n+1$ through $2 n-1$ and let $B$ be the in phase bracelet created by the tiles covering cells 1 through $n$. This process is clearly reversible since the phase of the bracelet uniquely identifies whether it is generated by the first or second case.

While the last proof established a one-to-one correspondence between two sets of tilings, identity (4), (after replacing $n$ with $n+1$ ), which is equivalent to

$$
l_{n}+l_{n+2}=5 f_{n}
$$

will be established by a five-to-one correspondence. For an $n$-board tiling $T$, we generate five distinct bracelets of size $n$ or $n+2$. As Figure 7 illustrates, the first
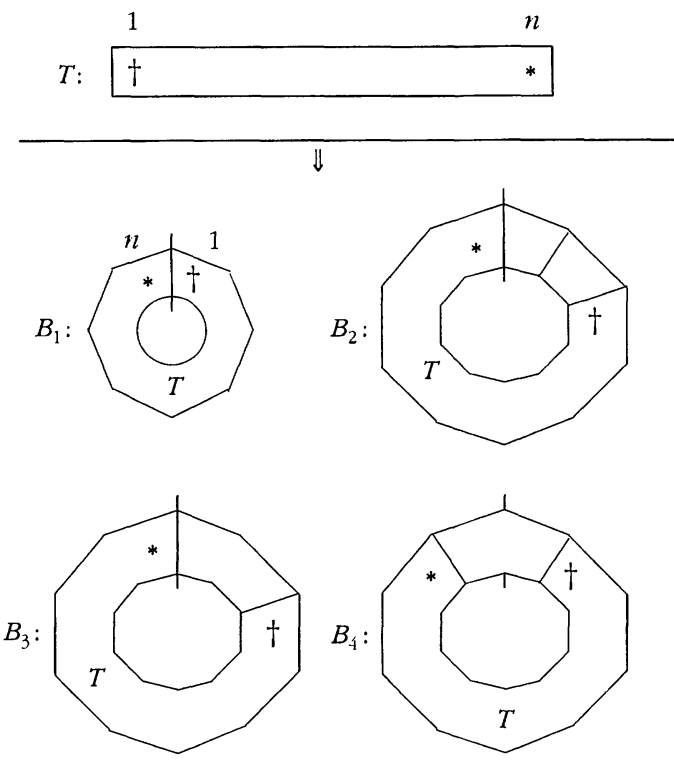

Figure 7. From a tiled $n$-board $T$, create an in phase $n$-bracelet and 3 distinct $(n+2)$ bracelets. 
four bracelets are:

$B_{1}$ : In phase $n$-bracelet obtained from $T$ by gluing cells $n$ and 1 together.

$B_{2}$ : In phase $(n+2)$-bracelet obtained by placing squares on cells 1 and 2 , followed by $T$.

$B_{3}$ : In phase $(n+2)$-bracelet obtained by placing a domino on cells 1 and 2 , followed by $T$.

$B_{4}$ : Out of phase $(n+2)$-bracelet obtained by placing a domino on cells $n+2$ and 1 , followed by $T$.

At this point in our construction, there are only two types of bracelets that have not been accounted for, namely: out of phase $n$-bracelets and in phase $(n+2)$-bracelets that begin with a square followed by a domino. Our last bracelet $B_{5}$ will depend on whether $T$ ends with a square or domino. See Figure 8.
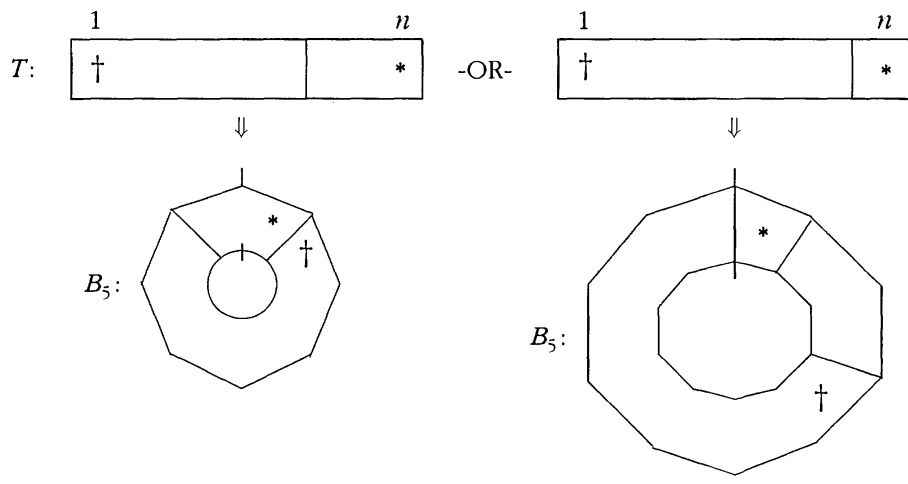

Figure 8. The fifth bracelet created from $T$ depends on whether $T$ ends with a domino or a square. This completes the five-to-one correspondence to prove $l_{n}+l_{n+2}=5 f_{n}$.

$B_{5}$ : If $T$ ends with a domino, then $B_{5}$ is the out of phase $n$-bracelet obtained by rotating the tiles of $B_{1}$ clockwise one cell.

If $T$ ends with a square, then $B_{5}$ is the in phase $(n+2)$-bracelet obtained by rotating the tiles of $B_{3}$ clockwise one cell.

This completes the desired five-to-one correspondence.

\section{Simultaneous Tilings}

At first glance, identity (6) does not look amenable to combinatorial interpretation due to the presence of the $(-1)^{n}$. An approach using simultaneous tilings and induction was successfully used in [6]. Here we draw a more direct connection by exhibiting an almost one-to-one correspondence which does the trick.

In this section, it will be convenient to establish a descriptive notation for tiling an $n$-board. We shall $\cdot$ let $d^{e_{1}} s^{e_{2}} d^{e_{3}} s^{e_{4}} \cdots$ denote the tiling beginning with $e_{1} \geq 0$ dominoes, followed by $e_{2} \geq 1$ squares, then $e_{3} \geq 1$ dominoes, and so on, where $2 e_{1}+e_{2}+2 e_{3}+e_{4}+\cdots=n$. For example, the tiling in Figure 2 is $d s d^{2} s d s$.

By the standard transformation, identity (6) becomes

$$
f_{n}^{2}=f_{n-1} f_{n+1}+(-1)^{n} \text {. }
$$

The left hand side of our identity counts the number of ordered pairs $(A, B)$, where $A$ and $B$ are both tilings of $n$-boards. The $f_{n-1} f_{n+1}$ term on the right hand side 
counts the number of ordered pairs $\left(A^{\prime}, B^{\prime}\right)$, where $A^{\prime}$ and $B^{\prime}$ are tilings of an $(n-1)$-board and an $(n+1)$-board, respectively. Given a simultaneous tiling ( $A, B$ ) not equal to an all-domino tiling, there exists a unique $k, 0 \leq k<n / 2$, such that the first $k$ tiles of both $A$ and $B$ are dominoes, but the $k+1$ st tile of $A$ or $B$ is a square. See Figure 9. Suppose the $k+1$ st tile of $A$ is a square, then $A=d^{k} s a$ where $a$ is a tiling of length $n-2 k-1$, and $B=d^{k} b$, where $b$ is a tiling of length $n-2 k$. Here we associate the pair $\left(A^{\prime}, B^{\prime}\right)$, where $A^{\prime}=d^{k} a$ and $B^{\prime}=d^{k} s b$. On the other hand, if the $k+1$ st tile of $A$ is a domino, then $A=d^{k+1} a$ where $a$ has length $n-2 k-2$, and $B=d^{k} s b$, where $b$ has length $n-2 k-1$. In this case, we associate the pair $\left(A^{\prime}, B^{\prime}\right)$, where $A^{\prime}=d^{k} s a$ and $B^{\prime}=d^{k+1} b$. Notice that for both $(A, B)$ and $\left(A^{\prime}, B^{\prime}\right)$, the first square occurs in cell $2 k+1$, i.e., $k$ is preserved in our mapping. The reverse mapping is constructed by essentially letting $B^{\prime}$ play the role of $A$ and $A^{\prime}$ play the role of $B$. When $n$ is even, the pair $\left(d^{n / 2}, d^{n / 2}\right)$ has no first square, and is therefore not paired up with any $\left(A^{\prime}, B^{\prime}\right)$. Thus, for even $n$, $f_{n}^{2}=f_{n-1} f_{n+1}+1$. When $n$ is odd, then $(A, B)$ necessarily has a first square, but will never be paired with $\left(d^{(n-1) / 2}, d^{(n+1) / 2}\right)$. Thus, for odd $n, f_{n}^{2}=f_{n-1} f_{n+1}-1$.

Case 1:

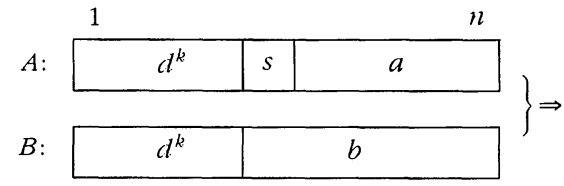

$A^{\prime}:$

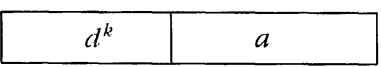

$B^{\prime}$ :

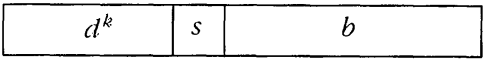

Case 2:

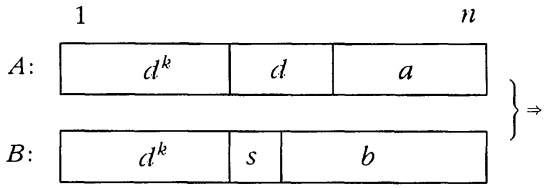

$A^{\prime}$ :

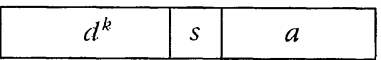

$B^{\prime}$

\begin{tabular}{|l|l|l|}
\hline$d^{k}$ & $d$ & $b$ \\
\hline
\end{tabular}

Figure 9. To prove $f_{n}^{2}=f_{n-1} f_{n+1}+(-1)^{n}$, we draw an almost one-to-one mapping between $n$-board tiling pairs and pairs of $(n-1)$-board and $(n+1)$-board tilings.

It is not surprising that there exists an analogous Lucas version of identity 6 . Using a similar argument, one can show that

$$
l_{n}^{2}=l_{n-1} l_{n+1}+(-1)^{n} \cdot 5
$$

For a combinatorial proof of this and more general identities see [3].

\section{Concluding Remarks}

So where do you go from here? We have merely scratched the surface of the numerous Fibonacci and Lucas identities that exist. More complicated identities can be proved combinatorially by introducing colored tilings or binary sequences [2, 3]. What about other Fibonacci-like sequences such as 5, 9, 14, 23, 37, 60, ..? We can show that the $n$th term of this sequence counts the number of Fibonacci tilings of length $n$ that can begin with either a domino in five possible phases or a square in nine possible phases. This leads to combinatorial proofs for generalized Fibonacci identities [3].

We leave the reader with some open questions. Can tilings be used to explain identities involving other recursively generated sequences? Can tilings with different 
objects or on different surfaces be used to discover further identities? Are there combinatorial interpretations of Fibonacci identities which use real numbers such as $F_{n}=\left(\left(\frac{1+\sqrt{5}}{2}\right)^{n}-\left(\frac{1-\sqrt{5}}{2}\right)^{n}\right) / \sqrt{5}$ ? (News flash! Yes! See [5]. Recently, tiling has even been used to "explain" continued fraction identities [4].) The possibilities are practically uncountable.

\section{References}

1. S. L. Basin and V. E. Hoggatt, Jr., A primer on the Fibonacci sequence, Part I, Fibonacci Quarterly, 1:1(1963) 65-72.

2. A. T. Benjamin, and J. J. Quinn, Fibonacci and Lucas identities through colored tilings, to appear in Utilitas Arithmetica.

3. A. T. Benjamin, J. J. Quinn, and F. E. Su, Recounting generalized Fibonacci identities, to appear in Fibonacci Quarterly.

4. A. T. Benjamin, J. J. Quinn, and F. E. Su, Counting on continued fractions, preprint.

5. A. T. Benjamin, K. Mahlburg, and J. J. Quinn, Random Fibonacci identities, preprint.

6. R. C. Brigham, R. M. Caron, P. Z. Chinn, and R. P. Grimaldi, A tiling scheme for the Fibonacci numbers, J. Recreational Math., 28:1 (1996 - 97) 10-16.

7. R. B. Nelsen, Proof Without Words: Exercises in Visual Thinking, Mathematical Association of America, 1993.

8. H. Prodinger and R. F. Tichy, Fibonacci numbers of graphs, Fibonacci Quarterly, 20 (1982) 16-21.

9. W. Staton and C. Wingard, Independent sets and the golden ratio, this JOURNAL, 26:4 (1995) 292-296

10. S. Vajda, Fibonacci \& Lucas Numbers, and the Golden Section: Theory and Applications, John Wiley and Sons, 1989.

11. R. M. Young, Excursions in Calculus, Mathematical Association of America, 1992, Chapter 3.

\section{Approximating Volumes}

From page 40 of Catalog A-20 (no date) from Paradise Water Gardens, 14 May Street, Whitman, Massachusetts 02382:

\section{Important Information}

Formula for calculating number of gallons of water contained in a pool. Because most pools are irregular, the capacity will be + or - based on various contours within a rectangle or square (in gallons).

Depth $\times$ length $\times$ width $\times 7.5$ gallons.

Circular - diameter $\times$ diameter $\times$ depth $\times 5.9$ gallons

Oval - width $\times$ length $\times$ depth $\times 6.7$ gallons.

If there are 7.5 gallons in a cubic foot of water, the first formula is correct. So is the second, since the number of gallons of water in a right circular cylinder with base radius $r$, diameter $d$, and depth $b$ is

$$
7.5 \pi r^{2} b=7.5 \pi(d / 2)^{2} b=7.5(\pi / 4) d^{2} b=d \times d \times b \times 5.9 .
$$

But where does that 6.7 in the last formula come from? For what sort of oval is the formula exact? 